\title{
Anti-Amnesic Activity of Vitex Negundo in Scopolamine Induced Amnesia in Rats
}

\author{
Abhinav Kanwal, ${ }^{1,2}$, Jogender Mehla ${ }^{3}$, Madhusudana Kuncha, Vegi Ganga Modi Naidu, \\ Yogendra Kumar Gupta ${ }^{3}$, Ramakrishna Sistla ${ }^{1 *}$
}

${ }^{1}$ Division of Pharmacology, Indian Institute of Chemical Technology (IICT), Hyderabad, India; ${ }^{2}$ National Institute of Pharmaceutical Education and Research (NIPER), Hyderabad, India; ${ }^{3}$ Department of Pharmacology, All India Institute of Medical Sciences (AIIMS), New Delhi, India.

Email: sistla@iict.res.in

Received June $8^{\text {th }}, 2010$; accepted July $12^{\text {th }}, 2010$.

\begin{abstract}
In the present study we investigated the anti-amnesic activity of Vitex negundo in scopolamine induced amnesia in rats. Wistar rats (180-200 g) were trained on active avoidance task. Each animal received session of 15 trials with inter trial duration of $15 \mathrm{~s}$ for 5 days. Scopolamine ( $3 \mathrm{mg} / \mathrm{kg}$, i.p) was administered at different time periods on the basis of stages of memory i.e acquisition, consolidation and retention in different groups $(n=6)$. Effect of Vitex negundo extract was evaluated and compared to a standard drug, Donepezil. Significant $(p<0.05)$ increase in the avoidance response on the $5^{\text {th }}$ session has been observed as compared to $1^{\text {st }}$ session in control group. Scopolamine treatment significantly $(p<$ $0.05)$ reduced the avoidance response compared to control. Extract treated groups shown significant $(p<0.05)$ increase in number of avoidance responses as compared to scopolamine treated groups. Increased oxidative stress in brain after scopolamine treatment, as observed by increase in MDA \& decrease in GSH \& SOD, was lowered in the groups treated with extracts. AChE activity was also improved after $V$. negundo treatment. Results of the study have shown that $V$. negundo treated groups decrease the phenomenon of amnesia by increasing learning of memory through antioxidant effect and decreasing AChE activity.
\end{abstract}

Keywords: Vitex Negundo, Amnesia, Acetylcholinestrase, Scopolamine, Learning and Memory, Oxidative Stress

\section{Introduction}

The Memory is the most important function of the brain. Memory is the process by which organisms are able to record their experiences and use this information to adapt their responses to the environment. Hence it is vital for survival [1]. Central cholinergic system is considered as the most important neurotransmitter involved in regulation of cognitive functions [2]. Impaired cognitive functions are the major features of Alzheimer disease (AD) [3]. Presence of acetylcholine within the neocortex is sufficient to ameliorate learning deficits and restore memory [4]. The prevalence of AD increases with the age (65 yrs) from $2 \%$ to $30-45 \%$ in those over 85 yrs [5]. $\mathrm{AD}$ and stroke together rank as the third most common causes of death [6]. The incidence of AD for those aged 65 yrs and older was 3.24 per 1000 individuals in a year [7]. One study in India showed that, the median survival time determined to be 3.3 yrs for patients with dementia and 2.7 yrs for patients with AD [8]. Scopolamine, a nonselective muscarinic cholinergic antagonist, is a wellknown centrally acting cholinergic probe, which causes impairment in learning [9]. In addition, scopolamine also causes increase in cognitive impairment in healthy elderly subjects compared to young adults [10]. The treatment with AChE inhibitors and muscarinic receptors agonists which increases cholinergic neurotransmission causes an improvement in cognitive deficits in AD [11]. Besides reducing cholinergic activity, oxidative stress plays an important role and is one of the major causes for memory loss in $\mathrm{AD}[12,13]$

Extensive research is going on different plants all around the world as plant extracts have a relatively higher therapeutic window, lesser side effects and are economical. Plant extracts may also provide a source of new compound as many synthetic drugs have been originated from herbal sources. Vitex negundo, a deciduous shrub belonging to family Verbenaceae that comprises 75 genera and nearly 2500 species, chiefly occurs in Pakistan, India and Srilanka. Though almost all 
parts of the plant are used, the extract from leaves and the roots is the most important in the field of phytomedicine and is sold as drugs. The leaf extract is used in Ayurvedic and Unani system of medicine [14]. Water extract of mature fresh leaves exhibited anti-inflammatory, analgesic and antihistamine properties [15]. Literature survey of $V$. negundo revealed the presence of volatile oil, triterpenes, diterpenes, sesquiterpenes, lignan, flavonoids, flavones glycosides, iridoid glycosides, and stilbene derivative [14]. Lignans, one class of natural compounds present in $V$. negundo, showed anti-cholinesterase activity in in-vitro [14]. However no studies were conducted to explore the effect of $V$. negundo extract against memory impairment in in-vivo.

In the process of learning and memory, three important stages have been suggested viz., acquisition, consolidation and recall of the learned task [16]. The Scopolamine hydrobromide is an anticholinergic drug, which produces amnesia by reducing the levels of acetylcholine, which is considered to be an important neurotransmitter for the learning and memory. Therefore, the present study was aimed to investigate the anti-amnesic effect of $V$. negundo aqueous extract on scopolamine administered at different stages of active avoidance learning in rats.

\section{Materials and Methods}

\subsection{Materials}

Aqueous extract of the plant Vitex negundo was obtained from Amruta herbals Pvt Limited, Indore (M.P), India, (Batch no. AHVN/556.) along with the copy of certificate of analysis. Scopolamine hydrobromide, Thiobarbituric acid (TBA), Glutathione, DTNB, Acetylthiocholine all were purchased from Sigma-Aldrich (Bangalore, India). SOD kit was purchased from Fluka. Other chemical and reagents are of analytical grade.

\subsection{Animals}

Male Wistar rats weighing between 180-200 g were obtained from National Institute of Nutrition, Hyderabad. The animals were housed in an animal facility of Indian Institute of Chemical Technology (IICT). The animal house maintained at $20 \pm 2^{\circ} \mathrm{C}$ and $50-60 \%$ relative humidity. A 12-hour dark/light cycle was maintained throughhout the study. Air changes were maintained with $5 \mu$ HEPA filter. Rats had free access to food (pellet diet supplied from M/s Petcare India Ltd., Bangalore) and water ad libitum. This study protocol was approved by the Institutional Animal Ethics Committee of Indian Institute of Chemical Technology, Hyderabad.

\subsection{Behavioral Test}

\subsubsection{Two-Way Active Avoidance with Negative (Punishment) Reinforcement}

The animals were trained on Active Avoidance Task in an automatic reflex conditioner with two-way shuttle box (Ugo Basile, Italy). The rats were treated orally with the standard drug through an intragastric feeding tube. Similarly the plant extract were administered for 14 days. For this purpose each rat is placed in a compartment separated from the other one by a guillotine door in the shuttle box. Exploration period of $2 \mathrm{~min}$ is given initially. There after, the trial start. In each trial the animal is subjected to a light for $30 \mathrm{~s}$ followed by a sound stimulus for $10 \mathrm{~s}$. Immediately after the sound stimulus, the rat receives a single low intensity foot shock $(0.5 \mathrm{~mA} ; 3 \mathrm{~s})$ from $10^{\text {th }}$ day to $14^{\text {th }}$ through the floor grid if it does not transfer to the other shock free compartment. Infrared sensors monitor the transfer time from one compartment to another, which is recorded as avoid (after the stimulus of either light alone or both light and sound) and escape (after the foot shock) response. Each animal received a daily session of 15 trials with an inter-trial duration of 15 $\mathrm{s}$ for 5 days i.e., a maximum of 75 trials. The rats were evaluated on the basis of their performance in the last session i.e., in the $5^{\text {th }}$ session for their decrease in amnesic activity and increased learning and memory. The criterion for improved cognitive activity was taken as significant increase in the avoidance response on $5^{\text {th }}$ session (retention) compared to $1^{\text {st }}$ session.

\subsection{Scopolamine Induced Loss of Memory in Rat}

Acquisition: scopolamine was administered 5 min prior to $1^{\text {st }}$ Trial on $1^{\text {st }}$ session

Consolidation: scopolamine was administered $5 \mathrm{~min}$ after the $15^{\text {th }}$ (i.e., last) trial on $1^{\text {st }}$ session (Training session)

Retention: scopolamine was administered 5 min prior to the $1^{\text {st }}$ trial on the last session i.e., $5^{\text {th }}$ session (Training session)

Dementia effect of scopolamine was evaluated on the basis of significant decrease in number of avoidance response in the treated groups as compared to that of control group in the last session i.e., $5^{\text {th }}$ session.

\subsection{Treatment Schedule}

The animals were divided into eight different groups $(\mathrm{n}=$ 6). Scopolamine (3 mg/kg, i.p) was administered at different time periods in the three groups (GR-2, GR-3, and GR-4) as follows:

Group I (GR-1)-Saline (control),

Group II (GR-2)-scopolamine was administered $5 \mathrm{~min}$ prior to $1^{\text {st }}$ Trial on 1st session (Training session)

Group III (GR-3)-scopolamine was administered 5 min after the $15^{\text {th }}$ (i.e., last) trial on $1^{\text {st }}$ session (Training session).

Group IV (GR-4)-scopolamine was administered 5 min prior to the $1^{\text {st }}$ trial on the last session i.e., $5^{\text {th }}$ session.

Group V (GR-5)-Standard drug, Donepezil (5 mg/kg) 
is given to GR-2 rats prior to 1 hour of $1^{\text {st }}$ trial.

Group VI (GR-6)-Similar to GR-2 but rats were pre-treated with plant extract for 14 days.

Group VII (GR-7)-Similar to GR-3 but rats were pre-treated with plant extract for 14 days.

Group VIII (GR-8)-Similar to GR-4 but rats were pretreated with plant extract for 14 days.

\subsection{Biochemical Estimation of Markers of Oxidative Stress}

On day $14^{\text {th }}$ following the behavioral testing, animals were sacrificed and the brain tissues were quickly removed, cleaned with ice-cold saline and stored at $-80^{\circ} \mathrm{C}$ for biochemical estimation.

\subsubsection{Preparation of Brain Homogenate}

Brain-tissue samples were thawed and homogenized with 10 times (w/v) ice-cold 0.1 M phosphate buffer ( $\mathrm{pH}$ 7.4). Aliquots of homogenates from the rat brains were separated and used to measure protein, lipid peroxidation and glutathione. The remaining homogenates were centrifuged at 10,000 rpm for $15 \mathrm{~min}$ and the supernatant was then used for enzyme assay. Superoxide dismutase was determined within $24 \mathrm{~h}$.

\subsubsection{Estimation of Malondialdehyde (MDA)}

Aliquotes of $0.5 \mathrm{ml}$ distilled water and $1.0 \mathrm{ml} \mathrm{10 \%} \mathrm{TCA}$ were added to a volume of $0.5 \mathrm{ml}$ brain tissue homogenate, mixed well and centrifuged at $3000 \mathrm{rpm}$ for $10 \mathrm{~min}$. To $0.2 \mathrm{ml}$ supernatant, $0.1 \mathrm{ml}$ thiobarbituric acid (TBA) $(0.375 \%)$ was added. The total solution was placed in a water bath at $80^{\circ} \mathrm{C}$ for $40 \mathrm{~min}$ and then cooled to room temperature. The absorbance of the clear supernatant was measured at $532 \mathrm{~nm}$ in spectrophotometer [17].

\subsubsection{Estimation of Superoxide Dismutase (SOD)}

The SOD activity of the brain tissue was analyzed by using the SOD Assay kit (Fluka). For the assay, $200 \mu$ of working solution, $20 \mu \mathrm{l}$ of dilution buffer and $20 \mu \mathrm{l}$ of enzyme working solution was added. Incubate the plate at $37^{\circ} \mathrm{C}$ for $20 \mathrm{~min}$. Absorbance was read at $450 \mathrm{~nm}$ using a microplate reader.

\subsubsection{Measurement of Glutathione}

Pipette out $100 \mu \mathrm{l}$ of the brain supernatant and add $50 \mu \mathrm{l}$ of O-ophthaldehyde $(100 \mu \mathrm{l} / \mathrm{ml})$. Incubate at room temperature for $15 \mathrm{~min}$. The flouroscent complex formed was read at an excitation wavelength of $350 \mathrm{~nm}$ and emission wavelength of $420 \mathrm{~nm}$ [18].

\subsubsection{Estimation of Cholinergic Status in the Rat Brain} The cholinergic marker, acetylcholinesterase was estimated in the whole brain according to the method of [19]. Briefly, the brains of the rats were removed over ice and the brain was separated using fine forceps. The tissue was then homogenized in $100 \mathrm{mM}$ phosphate buffer. 0.1 $\mathrm{ml}$ of this homogenate was incubated for 5 min with 2.7 $\mathrm{ml}$ of phosphate buffer and $0.1 \mathrm{ml}$ of DTNB. Then, 0.1 $\mathrm{ml}$ of freshly prepared acetylthiocholine iodide, $\mathrm{pH} 8$ was added and the absorbance was read at $412 \mathrm{~nm}$ for $3 \mathrm{~min}$ at $30,60,90,120,150$ and $180 \mathrm{sec}$.

\subsection{Statistical Analysis}

All data were expressed as mean $\pm \mathrm{SD}$. The significance of difference among the values of control, scopolamine treated, standard drug and extract treated groups for each session was determined by ANNOVA (one-way) followed by Dunnett's test. The difference between values on $1^{\text {st }}$ session and $5^{\text {th }}$ session of the same group was analyzed by student's t-test.

\section{Results}

\subsection{Selection of the Dose}

One single dose $(300 \mathrm{mg} / \mathrm{kg})$ of the herbal extract has been selected after the initial pilot study. This pilot study was done by taking limited number of Wistar rats. In the pilot study three different doses $(100,300 \& 900 \mathrm{mg} / \mathrm{kg})$ were taken. Based on initial data (data not shown) from active avoidance test $300 \mathrm{mg} / \mathrm{kg}$ was selected for the main study. It was also seen that animals with higher dose $(900 \mathrm{mg} / \mathrm{kg})$ tolerated the shock and remained at one place, which is not acceptable for the avoidance test. However, with lower dose $(100 \mathrm{mg} / \mathrm{kg})$ there was no significant difference in the number of avoidances between different groups of animals.

\subsection{Automatic Reflex Conditioner}

There was a significant $(\mathrm{p}<0.05)$ increase in avoidance response on $5^{\text {th }}$ session $(6.4 \pm 1.67)$ as compared to $1^{\text {st }}$ session $(3.0 \pm 1.00)$ in the control group (Table 1). All groups except GR-3 have shown significant $(p<0.05)$ increase in avoidance response compared to their first session data. Significant $(p<0.05)$ reduction of avoidance response was observed in scopolamine treated group (GR-2) compared to control group (GR-1). However standard drug (donepezil) treatment and extract feeding (GR-5 and GR-6) significantly $(\mathrm{p}<0.05)$ increased the avoidance response in their first session compared to their corresponding scopolamine treated group (GR-2). This reflects the effectiveness of donepezil as well as aqueous extract during scopolamine induced memory loss. However donepezil group (GR-5) showed improved response compared to extract treated group (GR-6) $(5.8 \pm 0.83$ vs $4.8 \pm 0.44)$ at the end of $5^{\text {th }}$ session. While extract treatment showed significant $(\mathrm{p}<$ 0.05 ) improvement of avoidance response at the end of $5^{\text {th }}$ session in GR-7, no improvement was observed in GR-8. 
Table 1. The number of avoidance responses in control (GR-1), scopolamine (GR-2, 3, 4) and drug treated (GR-6, 7, 8) groups (Mean $\pm \mathrm{SD}, \mathbf{n}=\mathbf{6}$ )

\begin{tabular}{ccccccc}
\hline S.NO. & GROUPS & DAY1 & DAY2 & DAY3 & DAY4 & DAY5 \\
\hline 1 & GR-1 & $03 \pm 1.00$ & $3.6 \pm 1.14$ & $4.2 \pm 0.83$ & $05 \pm 0.70$ & $6.4 \pm 1.67^{* *}$ \\
2 & GR-2 & $2.4 \pm 0.55$ & $03 \pm 1.00$ & $3.4 \pm 1.14$ & $3.8 \pm 0.83$ & $4.2 \pm 0.83^{* *, \dagger}$ \\
3 & GR-3 & $3.2 \pm 1.09$ & $2.6 \pm 0.54$ & $3.4 \pm 0.54$ & $3.4 \pm 0.89$ & $3.2 \pm 0.44$ \\
4 & GR-4 & $3.4 \pm 0.54$ & $3.6 \pm 0.89$ & $4.2 \pm 0.83$ & $4.6 \pm 0.54$ & $4.8 \pm 0.44^{*}$ \\
5 & GR-5 & $03 \pm 0.70$ & $04 \pm 100$ & $4.4 \pm 0.54$ & $5.4 \pm 0.89$ & $5.8 \pm 0.83^{* *, \Psi}$ \\
6 & GR-6 & $3.4 \pm 0.54$ & $3.8 \pm 0.83$ & $4.2 \pm 0.83$ & $4.8 \pm 0.83$ & $4.8 \pm 0.44^{* *,+}$, \\
7 & GR-7 & $3.6 \pm 0.54$ & $3.6 \pm 0.54$ & $3.8 \pm 0.44$ & $4.4 \pm 1.52$ & $4.6 \pm 0.54^{*, \Psi}$ \\
8 & GR-8 & $3.6 \pm 0.89$ & $3.8 \pm 0.44$ & $4.4 \pm 0.54$ & $4.8 \pm 0.44$ & $5.4 \pm 0.54^{* *}$
\end{tabular}

${ }^{*} \mathrm{p}<0.05$ vs. Day $1 ;{ }^{* *} \mathrm{p}<0.01$ vs. Day $1 ;{ }^{\dagger} \mathrm{p}<0.05$ vs. Standard drug $(\mathrm{GR}-6) ;{ }^{\Psi} \mathrm{p}<0.05$ vs. corresponding scopolamine treated group

\subsection{Markers of Oxidative Stress in Rat Brain}

\subsubsection{Malondialdehyde (MDA) levels}

Scopolamine treatment (GR-2, GR-3 and GR-4) significantly $(\mathrm{p}<0.05)$ increased the brain MDA level compared to control (GR-1) group (Figure 1). However only GR-3 showed significant $(\mathrm{p}<0.05)$ change compared to GR-1. Standard drug (GR-5) and aqueous extract of $V$. Negundo (GR-6, GR-7 and GR-8) treatment significantly $(\mathrm{p}<0.05)$ decreased brain MDA level compared to their corresponding scopolamine treated groups (GR-2, GR-3 and GR-4).

\subsubsection{Glutathione (Gsh) Levels}

Brain GSH level was decreased significantly $(\mathrm{p}<0.05)$ in scopolamine treated groups (GR-2, GR-3 and GR-4) compared to control (GR-1) (Figure 2). However standard drug (GR-5) and aqueous extract of $V$. Negundo (GR-6, GR-7 and GR-8) treatment significantly $(\mathrm{p}<0.05)$ increased brain GSH level compared to their corresponding scopolamine treated groups (GR-2, GR-3 and GR-4).

\subsubsection{SOD Activity}

SOD Activity has been expressed in \% inhibition rate. Scopolamine treatment decreased brain SOD activity significantly $(\mathrm{p}<0.05)$ in GR-2 and GR-3 groups but not in GR-4 (Figure 3). No improvement of SOD activity was observed in $V$. Negundo extract treated groups (GR-6, GR-7 and GR-8) compared to their corresponding scopolamine treated groups (GR-2, GR-3 and GR-4). However standard drug (GR-5) increased the SOD activity significantly $(\mathrm{p}<0.05)$ compared to the corresponding scopolamine treated group (GR-2).

\subsubsection{AChE Activity}

Acetylcholinestrase activity was estimated by the $\mathrm{V}_{\max }$ values as shown in Figure 4. The scopolamine treated groups have more $\mathrm{V}_{\max }$ values as compared to control group. Significant $(p<0.05)$ increased of AChE activity

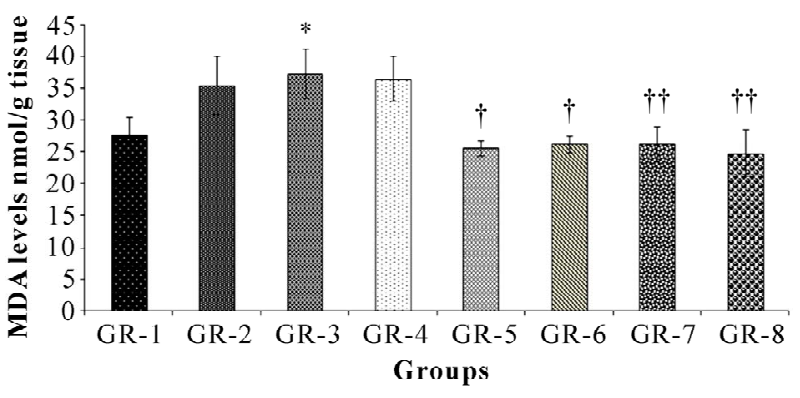

$* \mathrm{p}<0.05$ vs. control group

$\dagger \mathrm{p}<0.05$ vs. corresponding scopolamine treated group

$\dagger \dagger p<0.01$ vs. corresponding scopolamine treated group

Figure 1. Effect of aqueous extracts of Vitex Negundo (300 $\mathrm{mg} / \mathrm{kg}$ body wt.) on MDA levels in brains on Session 5 on different groups (Mean \pm SD, $\mathbf{n}=\mathbf{6}$ )

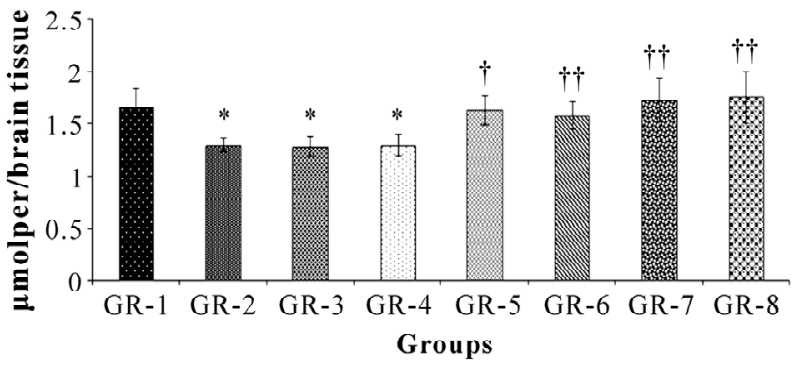

$* \mathrm{p}<0.05$ vs. control group

$\dagger \mathrm{p}<0.05$ vs. corresponding scopolamine treated group $\dagger \dagger p<0.01$ vs. corresponding scopolamine treated group

Figure 2. Effect of aqueous extracts of Vitex Negundo (300 $\mathrm{mg} / \mathrm{kg}$ body wt.) on Glutathione levels in brains on Session 5 on different groups (Mean $\pm \mathrm{SD}, \mathrm{n}=6$ ) 


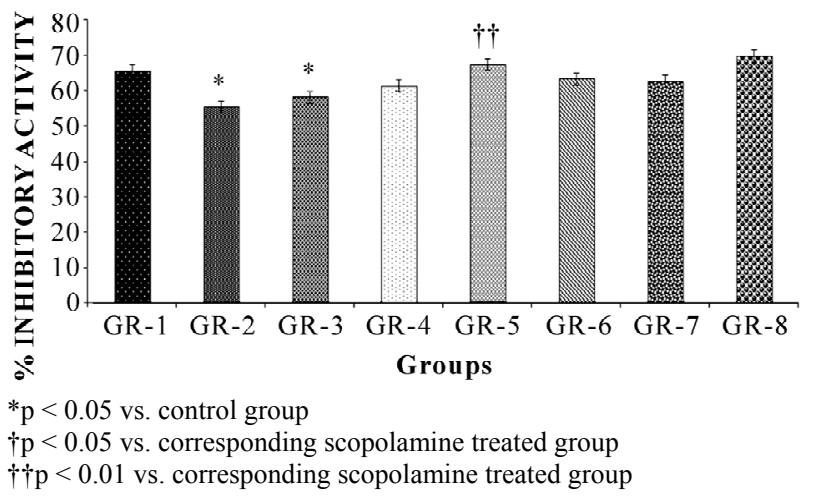

Figure 3. Effect of aqueous extracts of Vitex Negundo (300 $\mathrm{mg} / \mathrm{kg}$ body wt.) on SOD levels in brains on Session 5 on different groups (Mean \pm SD, $n=6$ )

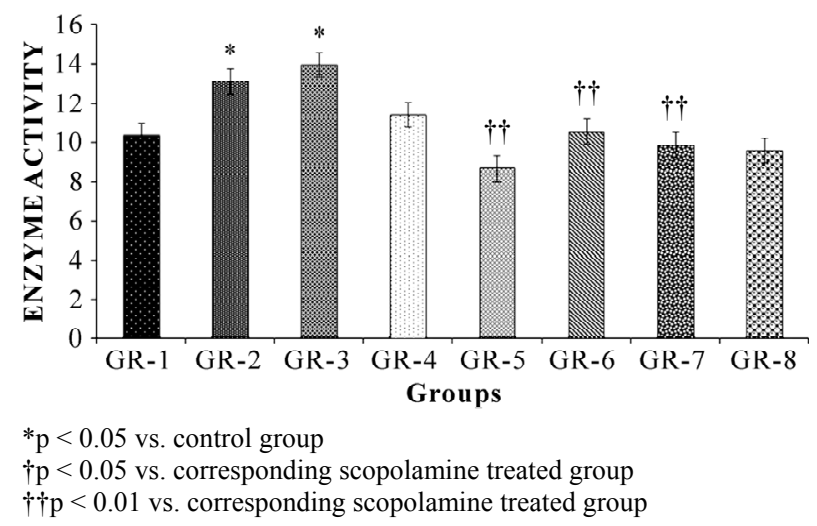

Figure 4. Effect of aqueous extracts of Vitex Negundo (300 $\mathrm{mg} / \mathrm{kg}$ body wt.) on AChE levels in brains on Session 5 on different groups (Mean \pm SD, $n=6$ )

was observed in scopolamine treated groups (GR-2 and GR-3, not in GR-4) compared to control (GR-1) (Figure 4). However standard drug (GR-5) and aqueous extract of $V$. Negundo (GR-6 and GR-7, not in GR-8) treatment significantly $(\mathrm{p}<0.01)$ decreased brain AChE activity compared to their corresponding scopolamine treated groups (GR-2, GR-3 and GR-4).

\section{Discussion}

$V$. negundo possesses many medicinal properties. Leaves of $V$. negundo have been investigated for its anti-inflammatory activity $[15,20]$. Telang et al first noticed non-steroidal anti-inflammatory (NSAID) activity of $V$. negundo. Similarly, fresh leaves of $V$. negundo have been suggested to possess anti-inflammatory and pain suppressing activities. Antinociceptive activity study of ethanolic leaf extract of $V$. negundo showed that it possesses both central and peripheral analgesic activity [21]. $V$. negundo has been also used in adjuvant therapy to standard anti-inflammatory drugs [22]. Literature survey of $V$. negundo also revealed the presence of lignans derivative, which is responsible for anti-cholinesterase ac- tivity in in-vitro [14]. $\mathrm{LD}_{50}$ dose of $V$. negundo leaf extract is $7.58 \mathrm{~g} / \mathrm{kg}$ which practically falls in the non- toxic dose range [23].

The administration of the antimuscarinic agent scopolamine produces transient memory deficit. Also, scopolamine has been shown to impair memory retention when given to rat shortly before training in an avoidance task. The ability of a range of different cholinergic agonist drugs to reverse the amnesic affects of scopolamine is now well documented in animals and human volunteers [24]. The scopolamine amnesia test is widely used as primary screening test for so called anti-Alzheimer drugs [24]. Here, scopolamine is given at different time of training sessions and trials. Such protocol is adapted to distinguish between the three different stages of memory i.e. acquisition, consolidation and retention.

In the preliminary screening of the present study showed that the improvement in learning and memory tasks in the shuttle-box was only observed at a dose of $300 \mathrm{mg} / \mathrm{kg}$ body wt. Therefore, the aqueous extract with $300 \mathrm{mg} / \mathrm{kg}$ was evaluated in more details. The avoidance responses shown by the animals were due to their ability to learn the task, which reflects the cognitive function. The task was investigated by using the scopolamine-induced dementia with the aqueous herbal extract of $V$. negundo. The animals were treated with scopolamine at different time intervals of trial in the sessions according to the different stages of the memory. Scopolamine administered $5 \mathrm{~min}$ prior to $1^{\text {st }}$ trial on $1^{\text {st }}$ session was for the acquisition whereas scopolamine administered 5 min after the $15^{\text {th }}$ (i.e., last) trial on $1^{\text {st }}$ session was for the consolidation stage of the memory. Similarly for the requisition (recall) the scopolamine was administered 5 min prior to the $1^{\text {st }}$ trial on the last session i.e., $5^{\text {th }}$ session. To evaluate the effect of the herbal aqueous extract of $V$. negundo, scopolamine was administered in the similar pattern in the pretreated herbal extract animals. The efficacy and potency of the extract was compared with the vehicle control and standard drug (Donepezil) group.

From the behavioral test i.e. two way shuttle box active avoidance test, it is clearly seen that there was a general decrease in the performance in the active avoidance in the scopolamine treated groups. The memory loss effect of scopolamine is more prominent compared to the control group. The aqueous herbal extract of $V$. negundo improved the memory loss effect of scopolamine in all three events like acquisition, consolidation and retention. As scopolamine-induced memory loss was more prominent in acquisition period, we administered standard drug, donepezil with this group. In comparison with Donepezil, the extract treated group had almost equal avoidance responses which indicates therapeutic efficacy of $V$. negundo against memory loss.

The present study therefore demonstrates the probable 
mechanism by which $V$. negundo enhanced the anti-amnesic activity by increasing the performance of learning and memory. It had been suggested that the varying degrees of behavioral impairments are associated with aging and age associated neurodegenerative diseases. Oxidative stress due to free radicals generation is responsible for producing the neuronal changes mediating these behavioral deficits [25]. Oxidative stress in brain generates oxygen radicals like superoxide anion, hydroxyl radical, and hydrogen peroxide, which act on polyunsaturated fatty acids in brain, thereby propagating the lipid peroxidation [26]. The major antioxidant and oxidative freeradical scavenging enzymes like glutathione, SOD and catalase plays an important role to reduce oxidation stress in brain. In the present study rats after scopolamine treatment showed a significant increase in the brain levels of malondialdehyde, which is the measure of lipid peroxidation and free radical generation. At the same time there was a significant reduction in levels of glutathione, a tripeptide found in all cells, which reacts with free radicals to protect cells from superoxide radical, hydroxyl radical and singlet oxygen [27]. Pre-treatment of $V$. negundo reduced the MDA levels and increased GSH content in brain after scopolamine treatment. Scopolamine reduced the SOD activity in brain. SOD is the only enzyme that uses the superoxide anions as the substrate and produces hydrogen peroxide as a metabolite. Super oxide anion is more toxic than $\mathrm{H}_{2} \mathrm{O}_{2}$ and has to be removed. Pretreatment with $V$. negundo significantly prevented the reduction of SOD activity in brain during scopolamine treatment. Our results also suggest that the aqueous extract of $V$. negundo reduced oxidative stress by reducing lipid peroxidation and increasing the endogenous antioxidant enzymes in brain. Other important activity has been shown by the extract is that it has acetylcholinetrase (AChE) inhibiting activity. This activity tends to allow the more retention of acetylcholine in the brain, which is important for the cognitive functions, learning and memory.

In conclusion, the present study demonstrates that aqueous $V$. negundo extract has potential therapeutic effects on improving the anti-amnesic activity in rats through inhibiting lipid peroxidation, augmenting endogenous antioxidant enzymes and decreasing acetylcholinestrase (AChE) activity in brain. Further study is warranted to find its potential use in humans.

\section{Aknowledgements}

Authors are very thankful to the Project Director, National Institute of Pharmaceutical Education and Research, Hyderabad and Director Indian Institute of Chemical Technology, Hyderabad for supporting this work. We are also thankful to Amruta Herbals Pvt Limited, Indore (M.P), India for providing the plant material.

\section{REFERENCES}

[1] J. Dunning and J. D. Matthew, "Molecular Mechanisms of Learning and Memory," Expert Reviews in Molecular Medicine, Vol. 5, No. 25, 2003, pp. 1-11.

[2] A. Blockland, "Acetylcholine: A Neurotransmitter for Learning and Memory?" Brain Research Review, Vol. 21, No. 3, 1996, pp. 285-300.

[3] M. F. Siddiqui and A. I. Levey, "Cholinergic Therapies in Alzheimer's Disease," Drugs of the Future, Vol. 24, No. 4, 1999, pp. 417-444.

[4] J. Winkler, S. Suhr and F. Gage, "Essential Role of Neocortical Acetylcholine in Spatial Memory," Nature, Vol. 375, No. 6531, 1995, pp. 484-487.

[5] T. F. Wernicke and F. M. Reischies, "Prevalence of Dementia in Old Age: Clinical Diagnoses in Subjects Aged 95 and Older," Neurology, Vol. 44, No. 2, 1994, pp. 250253.

[6] D. C. Ewbank, "Deaths Attributable to Alzheimer's Disease in the United States," American Journal of Public Health, Vol. 89, No. 1, 1991, pp. 90-92.

[7] V. Chandra, R. Pandav and H. H. Dodge, "Incidence of Alzheimer's Disease in a Rural Community in India: The Indo-US Study," Neurology, Vol. 57, No. 6, 2001, pp. 985-989.

[8] V. Chandra, M. Ganguli and R. Pandav, "Prevalence of Alzheimer's Disease and Other Dementias in Rural India: The Indo-US Study," Neurology, Vol. 51, No. 4, 1998, pp. 1000-1008.

[9] T. Sunderland, P. N. Tariot, R. M. Cohen, H. Weingarbier, E. A. Mueller and D. L. Murphy, "Anticholinergic Sensitivity in Patients with Dementia of the Alzheimer's Type and Age Matched Controls," Archives of General Psychiatry, Vol. 44, No. 5, 1987, pp. 418-426.

[10] C. Flicker, S. H. Ferris and M. Serby, "Hypersensitivity to scopolamine in the elderly," Psychopharmacology, Vol. 107, No. 2-3, 1992, pp. 437-441.

[11] E. Giacobini, "The Cholinergic System in Alzheimer's Disease," In: S. M. Aquilonius and P. G. Gillberg, (Eds.), Brain Research. Cholinergic Neurotransmission: Functional and Clinical Aspects, Elsevier, Amsterdam, 1990, pp. 321-332.

[12] W. R. Markesbery, "Oxidative stress hypothesis in Alzheimer's disease," Free Radical Biology and Medicine, Vol. 23, No. 1, 1997, pp. 134-147.

[13] M. A. Lovell, W. D. Ehmann, S. M. Butler and W. R. Markesberg, "Elevated Thiobarbituric Acid Reactive Substances and Antioxidant Enzyme Activity in the Brain in Alzheimer's Disease," Neurology, Vol. 45, No. 8, 1995, pp. 1594-1608.

[14] U. H. Azhar and M. Abdul, "Enzymes Inhibiting Lignans from Vitex Negundo," Chemical and Pharmaceutical Bulletin, Vol. 52, No. 11, 2004, pp. 1269-1272.

[15] M. G. Dharmasiri, J. R. Jayakodym, G. Galhenam, S. S. Liyanagem and W. D. Ratnasooriyam, "Anti Inflammatory and Analgesic Activities of Mature Fresh Leaves of Vitex Negundo," Jounal of Ethnopharmacology, Vol. 87, 
No. 2-3, 2003, pp. 199-206.

[16] A. C. Guyton and J. E. Hall, "Textbook of Medical Physiology," Harcourt Asia Pte Ltd, Singapore, 1999.

[17] H. Ohkawa, N. Ohishi and K. Yagi, "Assay for Lipid Peroxides in Animal Tissues by Thiobarbituric Acid Reaction," Analytical Biochemistry, Vol. 95, No. 2, 1979, pp. 351- 358 .

[18] P. J. Hissin and R. Hilf, "A Fluorometric Method for Determination of Oxidized and Reduced Glutathione in Tissues," Analytical Biochemistry, Vol. 74, No. 1, 1976, pp. 214-226.

[19] G. L. Ellman, D. K. Courtney, V. Andres and R. M. Featherstone, "A New and Rapid Colorimetric Determination of Acetylcholinesterase Activity," Biochemical Pharmacology, Vol.7, No. 2, 1961, pp. 88-95.

[20] R. S. Telang, S. Chatterjee and C. Varshneya, "Studies on Analgesic and Anti-Inflammatory Activities of Vitex Negundo Linn," Indian Journal of Pharmacology, Vol. 31, No. 5, 1999, pp. 363-366.

[21] R. K. Gupta and V. R. Tandon, "Antinociceptive Activity of Vitex-Negundo Linn Leaf Extract," Indian Journal of Pharmacology, Vol. 49, No. 2, 2005, pp. 163-170.
[22] R. K. Gupta and V. R. Tandon, "Vitex Negundo Linn (VN) Leaf Extract as an Adjuvant Therapy to Standard Anti-Inflammatory Drugs," The Indian Journal of Medical Research, Vol. 124, No. 4, 2006, pp. 447-450.

[23] M. N. Ghosh, "Fundamentals of Experimental Pharmacology," scientific book agency, Calcutta, 1984.

[24] M. H. V. Kumar and Y. K. Gupta, "Antioxidant Property of Celastrus Paniculatus Willd: A Possible Mechanism in Enhancing Cognition," Phytomedicine, Vol. 9, No. 4, 2002, pp. 302-311.

[25] C. I. Cantuti, B. Shukitt-Hale and J. A. Joseph, "Neurobehavioural Aspects of Antioxidants in Aging," International Journal of Developmental Neuroscience, Vol. 18, No. 4-5, 2000, pp. 367-381.

[26] T. Coyle and P. Puttfarcven, "Oxidative Stress, Glutamate and Neurodegenerative Disorder," Science, Vol. 262, No. 5134, 1993, pp. 89-695.

[27] J. B. Schulz, J. Linderau and J. Dichgans, "Glutathione, Oxidative Stress and Neurodegeneration," European Journal of Biochemistry, Vol. 267, No. 16, 2000, pp. 49044911 . 


\section{Graphical Abstract}

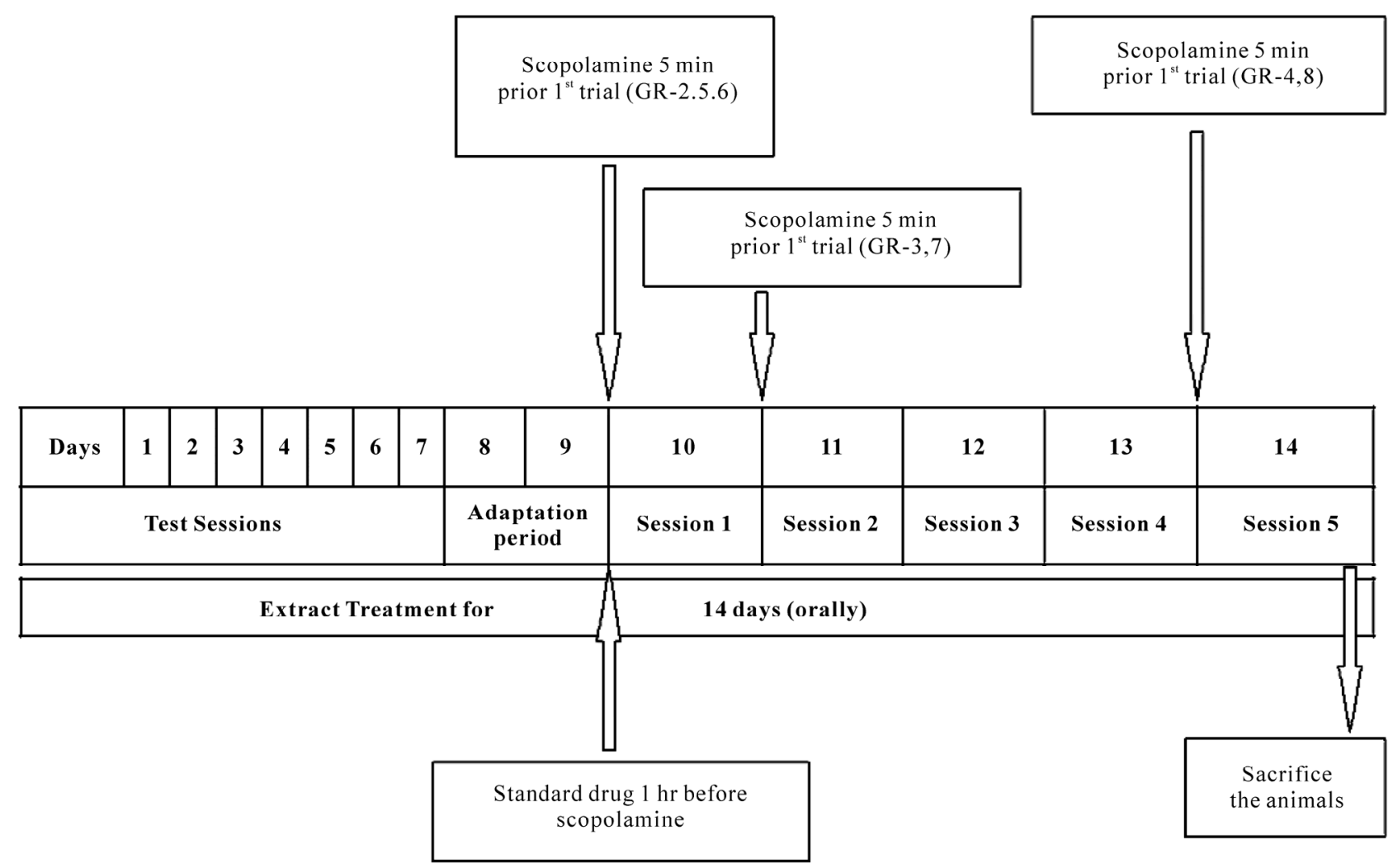

